\title{
Microporosity Quantification via NMR Relaxometry
}

\author{
Francisco Gallego-Gómez, ${ }^{*}$ Calin Cadar, Cefe López, Ioan Ardelean
}

\begin{abstract}
Micropores and their interplay with liquids are critical in many solid systems but challenging to investigate, often requiring complex and indirect techniques to even be detected. Here we perform low-field ${ }^{1} \mathrm{H}$ NMR transverse relaxometry on submicron Stöber silica spheres to directly detect micropore water. Colloidal crystals with a well-defined spheres configuration are used to facilitate unambiguous distinction of the interparticle voids water and enable straightforward micropore volume quantification. We evidence significant microporosity ( $\sim 16 \%$ of the sphere volume) with high water-accessibility and monitor the drying of the micropore water, which exhibits high resilience, evaporating only after complete voids drainage. Progressive microporosity closure by thermal annealing above $600{ }^{\circ} \mathrm{C}$ is shown, until complete removal at $900{ }^{\circ} \mathrm{C}$. Increasing hydrophobicity of the micropore walls is observed, although it barely affected the water access. Our results prove the capability of NMR relaxometry to quantitatively investigate complex microporosity and the behavior of water confined therein.
\end{abstract}

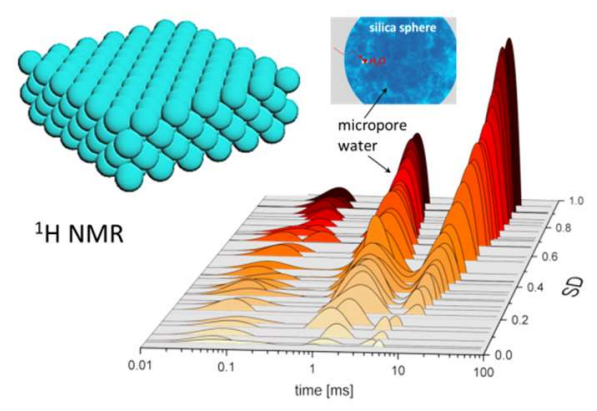

Porosity is a crucial aspect of many particulate systems and solids, as it determines essential material characteristics such as specific surface area, density, sorption volume, mechanical properties or refractive index. Thus, the detection and characterization of porosity still draws much attention. In particular, uncovering and analyzing microporosity (pore size $<2 \mathrm{~nm}$ ) represents a challenge in many cases. Even most standard characterization techniques such as adsorption isotherms ${ }^{[1]}$ may not detect complex or narrow microporosity due to kinetic restrictions for the probe molecules to access the pore volume. Besides, pore analysis by current methodologies, such as sorption and $\mathrm{x}$-ray or neutron diffraction techniques, usually require a number of assumptions that make the assignment of microporosity in multiporous media rather indirect and, often, inconclusive. Thus, more direct techniques for pore -and pore/fluid- research are needed, especially in the case of tortuous microporosity. In this sense, nuclear magnetic resonance (NMR) is a valuable, widelyused characterization technique of porous materials. ${ }^{[2]}$ In particular, NMR relaxometry determines the relaxation rate of nuclear spins in fluid molecules within the porous medium to study both the pore characteristics (dimensions, surface properties) and the confined fluid. ${ }^{[2]}$ However, despite its potential, NMR has barely been exploited for quantitative examination of microporosity, being its use often restricted to the identification of strongly interacting sites in a multiporous system. ${ }^{[3]}$ It must be noted that NMR cryoporosimetry, ${ }^{[4]}$ beside requiring more demanding equipment, is fundamentally limited for the study of small microporosity as water does not freeze in cavities of $\delta 1 \mathrm{~nm}$. In the present work, we demonstrate the capability of ${ }^{1} \mathrm{H}$ NMR relaxometry to directly detect -and discriminate- water located in complex microporosity, serving as a useful tool for quantitative

[] Dr. F. Gallego-Gómez, Prof. C. López.

Instituto de Ciencia de Materiales de Madrid (ICMM), Consejo Superior de Investigaciones Científicas (CSIC), Spain

Corresponding Author. E-mail address: francisco.gallego@icmm.csic.es Calin Cadar, Prof. Ioan Ardelean.

Department of Physics and Chemistry, Technical University of Cluj-Napoca, Romania investigation of both the micropores and the water confined therein.

As an adequate test system, we choose submicron Stöber silica spheres, ${ }^{[5]}$ which are the most-employed colloidal silica beads in many scientific and industrial fields, such as catalysis, ${ }^{[6]}$ life sciences, ${ }^{[7]}$ photonics, ${ }^{[8]}$ or nanomaterials. ${ }^{[9]}$ However, in spite of extensive applicability and intense research, structural aspects of Stöber spheres remain poorly understood, as is the case of their microporosity, and even its occurrence -and its accessibility to molecules such as water- still arouses controversy. Such a lack of knowledge must be ascribed to the complex nature of Stöber inner porosity, which hinders access of probe gas molecules, such as nitrogen, and precludes reliable textural description -and even detection- by standard techniques such as adsorption (see Ref. [10] and references therein). In addition, the inner particle porosity must be discriminated from the outer porosity, that is, the interparticle voids in the sample, usually in the form of powder or dense compacts; such distinction is often inefficient by e.g. thermogravimetry or infrared absorption. Hence, a Stöber sphere assembly constitutes a technologically relevant system of multiporous nature that involves intricate, poorly understood microporosity.

We performed low-field transverse NMR relaxometry on ordered monodisperse Stöber spheres building a solid colloidal crystal (CC). Two CCs (S1 and S2) were fabricated with spheres of diameter either $D=320$ or $260 \mathrm{~nm}$. The resulting ordered fcc spheres arrangement provided a well-defined interparticle void configuration, composed by tetrahedral and octahedral voids with equivalent sizes of $0.225 D$ and $0.414 D$, respectively, and a total volume fraction of $26 \%$ (in an ideal close-packed fcc sample). This a priori knowledge of both void size and volume in the sample offers a quantitative reference for straightforward correlation with other sites in the sample, if present, like the spheres inner porosity. Although still disputed, there is (rather indirect) evidence of the microporous character of Stöber silica. ${ }^{[11]}$ Recently, using combined isothermal experiments, microporosity in Stöber spheres of 245-300 $\mathrm{nm}$ has been quantified, ${ }^{10}$ allowing crosschecking with our results. Among the as-prepared CC samples (denoted as S1-0 and S2-0), annealed samples were also measured (see Experimental Section). Thermal annealing, which is known to enhance cross-linking of the silica network and gradually eliminate porosity, ${ }^{12}$ was performed to study its effect on 

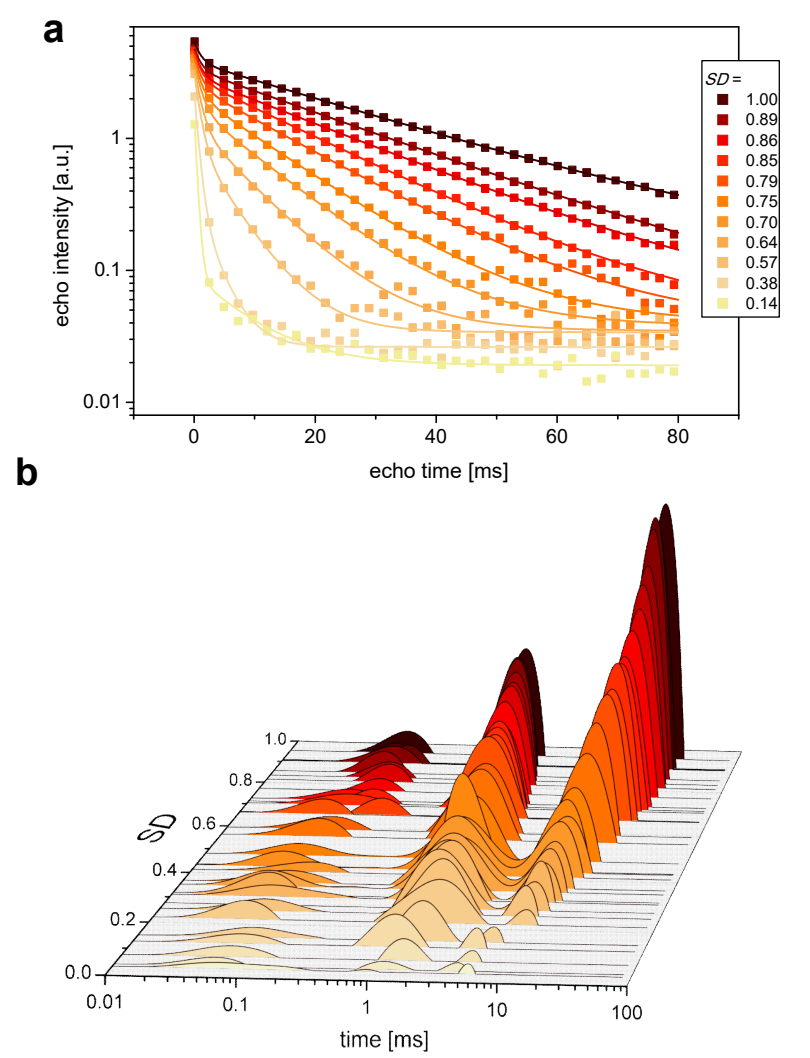

Figure 1 NMR transverse relaxation experiment during water-desaturation of the as-prepared S1-0 sample. (a) Experimental CPMG echo trains (symbols) and biexponential fits (lines). (b) Corresponding ILT relaxation time distribution.

Stöber microporosity. As-prepared pieces were subjected to four different temperatures $\left(600\right.$ and $800{ }^{\circ} \mathrm{C}$ on S2, 700 and $900{ }^{\circ} \mathrm{C}$ on S1), being the samples referred to as $\mathbf{S - 6 0 0}$ to $\mathbf{S - 9 0 0 . ~ H i g h e r ~}$ temperatures were avoided to prevent the occurrence of significant particles sintering, ${ }^{[12,13]}$ thus preserving the void morphology.

Sparse samples (ca. $200 \mathrm{mg}$ ) were used in order to assure the ordered arrangement of the spheres. Each sample was first measured in fully water-saturated conditions (saturation degree $S D=$ 1) and, then, successively during in situ drying until reaching a saturation degree $S D_{a}$ set by the ambient conditions in the NMR tube $\left(35^{\circ} \mathrm{C}\right.$ and $40 \%$ relative humidity). The wetting state at ambient conditions was considered, as it is relevant for practical interests. Further drying was achieved by heat- and vacuum-induced outgassing; the driest state is denoted as $S D_{\min }$. The transverse relaxation of the water molecules in the CCs was determined by a CPMG pulse sequence. ${ }^{[14]}$ Low-field NMR was employed to reduce diffusion effects on the echo trains. ${ }^{[15]}$ The effective transverse relaxation time $T_{2}$ of a liquid confined in a single pore of volume $V_{\text {pore }}$ can be approximated, in the saturated case (pore completely filled), by: $:^{[2 \mathrm{a}]}$

$$
T_{2} \cong T_{2 S} \frac{V_{\text {pore }}}{\lambda S_{\text {pore }}} \text {. }
$$

Here, $T_{2 \mathrm{~S}}$ is the transverse relaxation time of the liquid molecules in a fast-exchange surface layer, which is typically considered as a thin film of thickness $\lambda$ strongly bonded to the pore surface $S_{\text {pore }}$. Thus, Eq. (1) relates the relaxation time in a pore with its dimensions (ratio $V_{\text {pore }} / S_{\text {pore }}$ ) and corresponding $T_{2 \mathrm{~S}}$, which, in absence of paramagnetic centers in the sample, depends on the strength of the liquid-surface interaction. ${ }^{[2 \mathrm{a}]}$ In heterogeneous samples with e.g. multiple pore sizes, as ours, the recorded CPMG echo train will yield a multi-exponential decay, described by different relaxation times $T_{2}$ that can be extracted using a numerical inverse Laplace transform (ILT). ${ }^{[16]}$ Eq. (1) can then be used for direct comparison between the liquid contributions from different pore sizes: the smaller the pore, the faster the relaxation. Note, however, that Eq. (1) is not accurate for small pores (with size comparable to ג). Finally, in unsaturated pores $T_{2}$ decreases with decreasing pore filling. ${ }^{[3 \mathrm{c}, 17]}$

CPMG echo trains recorded in as-prepared samples approached bi-exponential decays (S1-0 data in Figure 1a), pointing to a predominant two-mode relaxation time distribution, as confirmed by the corresponding ILT (Figure 1b). The clear-cut discrimination of the two contributing modes over the entire drying experiment evidences the existence of water located in two types of confinements with well-differentiated morphologies and/or bonding conditions, each having a specific transverse relaxation time. On account of the abovementioned pore configuration in the $\mathrm{CC}$, the slower and faster modes are attributed to the water located in the interparticle voids and the inner sphere microporosity, respectively. Their specific relaxation times and volumes, given by the maxima and areas of the respective distribution peaks for each $S D$, will be referred to as $T_{2}{ }^{\text {void }}$, $T_{2}{ }^{\text {micro }}$ and $A^{\text {void }}, A^{\text {micro }}$. While the slow mode is studied in detail elsewhere (in the context of void imbibition and drainage of particulate packings), we focus here on the characterization of the Stöber microporosity via the fast mode. Note that, actually, a third, smaller peak systematically appeared at very short times $(\sim 0.1 \mathrm{~ms})$, which may be an artifact of the ILT; however, this peak could also correspond to tightly bound water molecules (e.g. structural water or water confined in very narrow micropores) - this residual contribution is not considered here.

Taking advantage of the known spheres arrangement in the $\mathrm{CC}$ (and, thus, known void characteristics), the relaxation time distribution at the fully saturated state $(S D=1$ in Figure 2 a) gives direct insight into the pores associated to the fast mode. On the one hand, $T_{2}{ }^{\text {micro }}(S D=1)=1.2 \mathrm{~ms}$ is 21 times smaller than $T_{2}{ }^{\text {void }}(S D=1)$, which, according to Eq. (1) and the void size (72-132 nm in S1-0), would lead to a small porosity dimensions of 3-6 nm. ${ }^{[18]}$ This estimate clearly surpasses the micropore size of $0.3-0.6 \mathrm{~nm}$ calculated from adsorption isotherms. ${ }^{[10]}$ However, as mentioned above, Eq. (1) cannot give an accurate estimate of pore sizes comparable to the surface layer thickness $\lambda$ (ca. $0.3 \mathrm{~nm}$, if a water monolayer is assumed), as in this case. Therefore, the ratio between relaxation times must be only considered as evidence that the fast mode is due to pores with size in the order of $1 \mathrm{~nm} \cdot{ }^{[19]}$ On the other hand, the peak area ratio $\left(A^{\mathrm{void}} / A^{\mathrm{micro}}\right)$ at full saturation corresponds to the volume ratio of the sample voids to the sphere micropores (those accessible to water molecules). Thus, the known void volume ( $26 \%$ of the whole sample) allows direct quantification of the microporosity: the measured peak area ratios were 2.16 and 2.24 in $\mathbf{S - 0}$ (for the 320and 260-nm samples, respectively) yield a micropore water volume $V_{\text {micro }}$ of 16.3 and $15.6 \%$ of the sphere volume. ${ }^{[20]}$ These significant volumes excellently agree with the water-accessed microporosity of $16 \%$ estimated from adsorption measurements. ${ }^{[10]}$ This simple quantitative analysis at full saturation allows to safely ascribe the fast relaxation peak to the water placed in the inner porosity of the spheres. Thereby, the use of an ordered particle assembly as the 

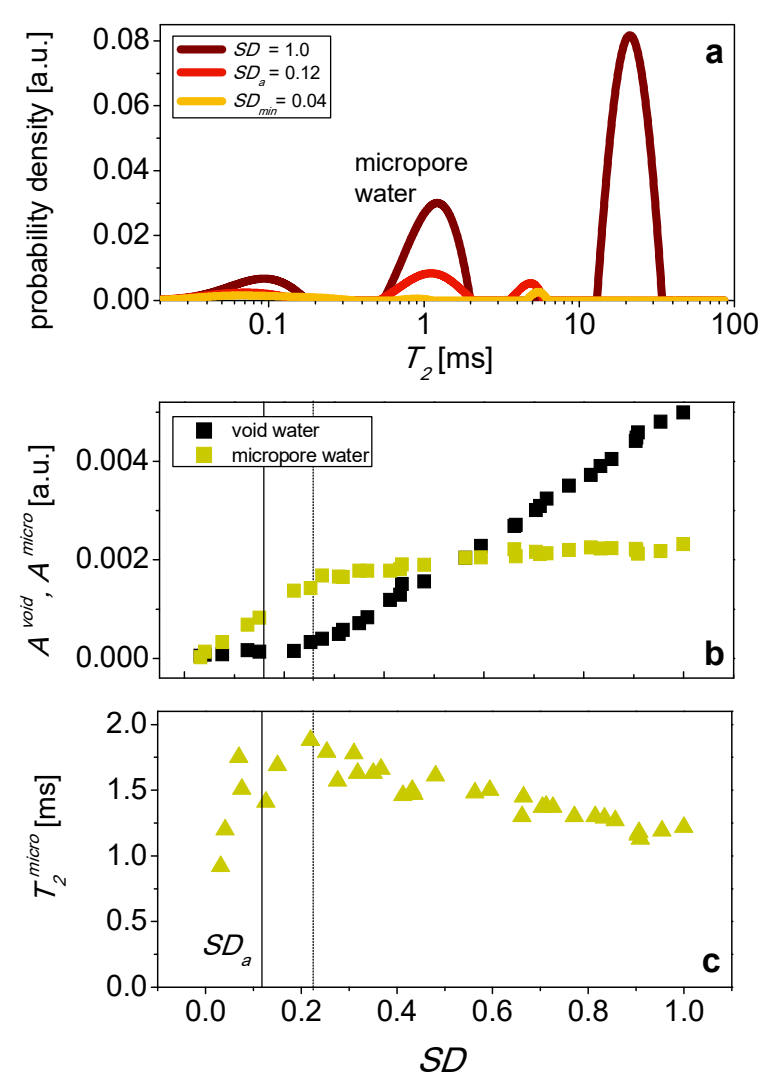

Figure 2 (a) Relaxation time distributions obtained in the as-prepared S1-0 sample at three characteristic saturation degrees (see text). (b) Evolution of the fast and slow mode areas, and (c) the fast mode relaxation time during water desaturation. The solid line denotes the saturation state under ambient conditions.

probe system constitutes a great advantage in order to avoid ambiguity in the identification and quantification of the microporosity. In fact, NMR relaxometry applied to porous systems cannot easily discriminate the different contributions of strongly confined or bonded water, the performance of micropore water, if present, remaining mostly indistinguishable. Here, the large water volume quantified as responsible for the fast mode discards its attribution to surface water adsorbed on the spheres (which would amount to less than 1 vol.\% in the CC geometry).

Since the different decay contributions remained well-defined along the sample drying experiment, the micropore draining could be 'monitored'. As a main feature, the micropore water was notably resilient and only decreased at high sample desaturation (Figure 1b). At equilibrium under the atmospheric conditions in the NMR tube, the relaxation time distribution virtually exhibited only fast relaxation mode $\left(S D_{a}=0.13\right.$, Figure $\left.2 \mathrm{a}\right)$ : that is, the sphere still held a significant amount of inner water-after a substantial decrease, though-, while practically no water remained outside. Upon outgassing, almost complete removal of the micropore water -vanishing fast relaxation mode- was achieved $\left(S D_{\min }=0.04\right.$, Figure $\left.2 \mathrm{a}\right) .{ }^{[21]}$ The micropore water drying, given by the fast mode evolution with $S D$, revealed two distinct regimes. On the one hand, $A^{\text {micro }}$ first remained almost constant but clearly decreased for $S D<0.25$ (Figure 2 b, green symbols). Remarkably, this performance appeared to directly depend on the progress of the void water (Figure $2 \mathrm{~b}$, black symbols): micropores begin to be evacuated significantly only when the voids are completely empty. This fact logically suggests that
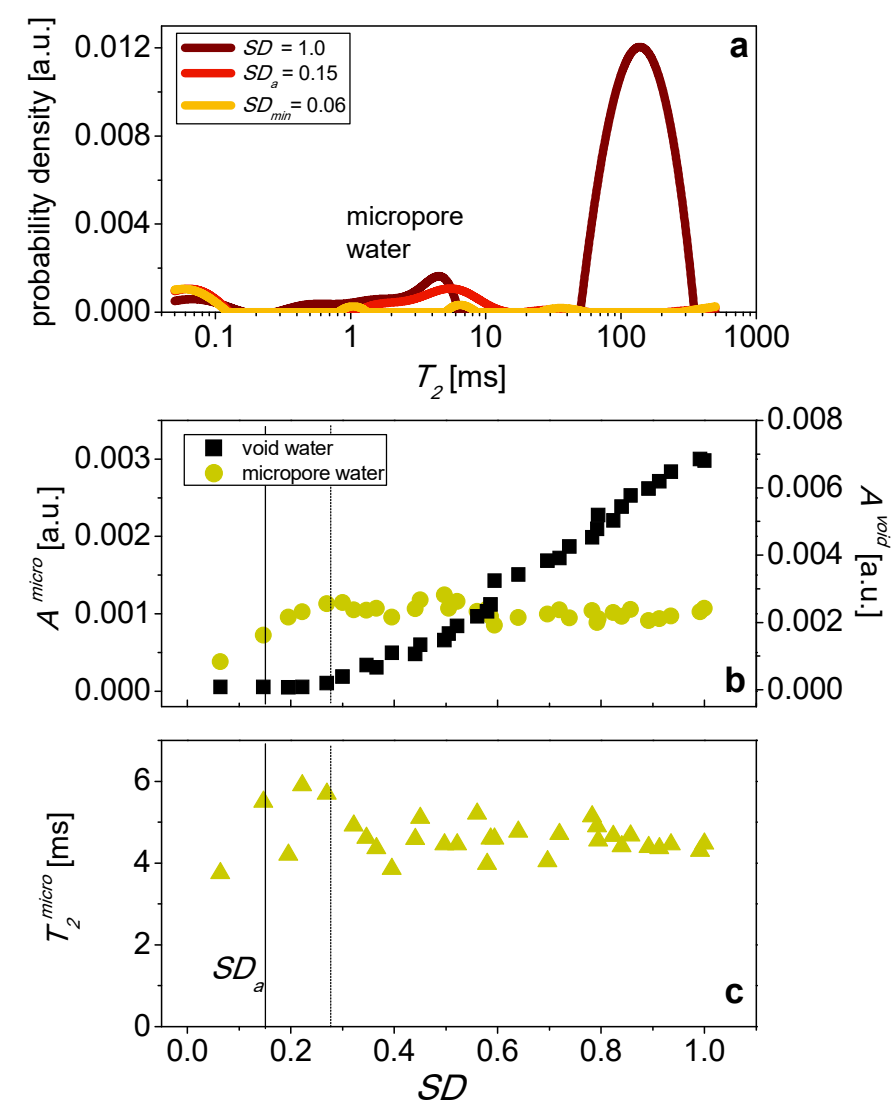

Figure 3 (a) Relaxation time distributions of the annealed S-700 sample at three characteristic saturation degrees. (b) Evolution of the fast and slow mode areas (left and right axes, respectively) and (c) the fast relaxation mode time during water desaturation. The solid line denotes the saturation state under ambient conditions.

the presence of water on the outer spheres surface precluded the evaporation from their inner porosity. On the other hand, $T_{2}{ }^{\text {micro }}$ also reflected a trend change at the same threshold (Figure 2c): while it varied only slightly during the first drying stage, it patently diminished concomitantly with $A^{\text {micro }}$, a fact that is ascribed to the progressive micropore evacuation.

From the monitoring of the micropore water through the fast relaxation mode, we directly make an important inference: water can efficiently access the Stöber sphere micropores and is mobile, that is, its amount evolves with the ambient conditions. ${ }^{[22]}$ This observation is nontrivial, as it has often been argued that micropore draining is very difficult ${ }^{[11 \mathrm{~b}, 11 \mathrm{c}, 12]}$ because of the strong capillary suction exerted in such narrow spaces, ever more in the case of hydrophilic pores. Optical characterization of artificial silica opals made of submicron Stöber spheres already pointed to easy variation of water content under changing ambient conditions; $;{ }^{[23]}$ however, no direct distinction between micropore and void water was possible, leading to inaccurate attribution in some cases. Further efforts for the assessment of micropore water in Stöber spheres have required combined techniques such as multi-adsorbate isotherms ${ }^{[10]}$ or simulation of opals photonic performance. ${ }^{[24]}$ Here, as the NMR experiment directly allows separate quantification of both micropore and void water contents, the variable filling of the Stöber microporosity is straightforwardly demonstrated. In particular, it is shown that, in unsaturated states, water preferentially fills the inner sphere volume (in accordance with adsorption ${ }^{[10]}$ and photonic experiments ${ }^{[24]}$ ). In equilibrium at ambient experimental conditions, the microporosity is only partially filled (also consistent with Refs. [10,24]) but still 


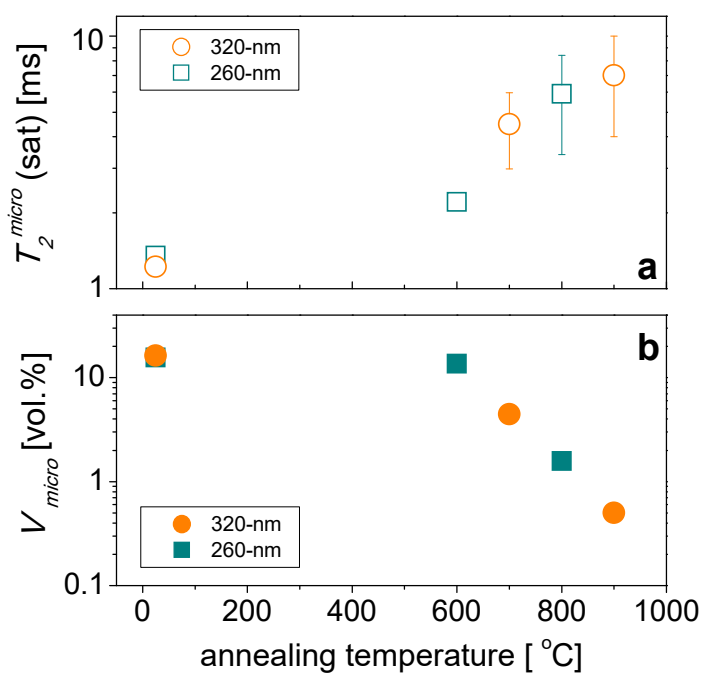

Figure 4 Evolution with the annealing temperature of characteristic micropore parameters at full saturation. (a) Fast mode relaxation time, and (b) micropore water volume (expressed in sphere vol.\%), calculated from the peak areas ratio.

retaining a remarkable amount of water: $4 \mathrm{wt} . \%$ of the dry sample or, when comparing with $A^{\text {micro }}$ at $S D=1$, about half the micropore volume.

Finally, we prove the capability of NMR relaxometry for direct investigation of the thermal effect on Stöber microporosity in quantitative terms of both pore volume and water behavior. While heat treatment is known to reduce the microporosity in Stöber spheres, its extent has been scarcely quantified (indirectly via e.g. specific area, density or refractive index $)^{[11 b, 13 b, 25]}$-and, sometimes, contradictorily; besides, the accessibility to the treated micropores has barely been studied. In the annealed CC samples, relaxometry experiments also led to well-distinguishable, predominantly two-mode relaxation time distributions (see S-700 representative data in Figure 3 as a typical case). However as a common trend, the fast mode split into several peaks, usually overlapping, which might reflect heterogeneity in the sphere microporosity induced by the thermal treatment. Though, the fast mode was still identifiable and easy to monitor, allowing reliable study of the micropore water. The drying behavior was found to be comparable to that in non-annealed samples. At the tube ambient conditions $\left(S D_{a}=0.15\right.$ in the case of S-700), the slow mode vanished again, meaning that water persisted only in the microporosity, being finally removed upon outgassing (Figure 3a). (Occasional narrow, small peaks appearing at low filling degrees were ILT artifacts). Interestingly, $A^{\text {micro }}$ at $S D_{a}$ was about half the full-saturation value (Figure $3 b$-the same was observed in $\mathbf{S - 6 0 0}$ and $\mathbf{S - 8 0 0}$ ), as in the non-annealed samples, suggesting a similar resilience. The evolution of $A^{\text {micro }}$ with $S D$ revealed again a threshold, confirming the interdependence with the presence of water in the voids: the micropore water began to dry only after full drainage of the voids (Figure $3 \mathrm{~b}$ ). $T_{2}{ }^{\text {micro }}$ remained constant down to the threshold, while the irregular peak shape precluded obtaining a clear trend at lower micropore fillings (Figure 3c).

While the thermal treatment had no drastic effect on the drying behavior of the micropore water, it clearly affected the Stöber sphere microporosity, as evidenced by the progression of both micropore volume and surface interaction (Figure 4). Therefore, as proceeded above, we consider the full saturated states to obtain, on the one hand, the fast mode relaxation time $T_{2}{ }^{\text {micro }}(S D=1)(\mathrm{de}-$ termined at the peak maximum); and, on the other hand, the micropore water volume $V_{\text {micro, }}$ as determined from the $A^{\text {void }} / A^{\text {micro }}$ ratio. Figure 4 a evidences the increase of $T_{2}{ }^{\text {micro }}(S D=1)$ with the annealing temperature -already at $600{ }^{\circ} \mathrm{C}$-, reflecting a weaker surface interaction with the water molecules and leading to longer $T_{2 \mathrm{~S}}$ in Eq. (1), which is attributable to the progressive silica dehydroxylation (-OH groups removal). ${ }^{[12,26]}$ Such behavior, also observed in the slow mode, demonstrates the thermal dehydroxylation of the micropore walls, in spite of being considered to occur with greater difficulty in inner surfaces. ${ }^{11 a}$ Finally, Figure $4 \mathrm{~b}$ shows a drastic decrease of the micropore water volume at $\geq 700{ }^{\circ} \mathrm{C}$, until virtual disappearance at $900{ }^{\circ} \mathrm{C}$. Obviously, $V_{\text {micro }}$ would not only be affected by the gradual microporosity collapse but also by a potentially lesser water-accessibility. However, the fact that the already noticeable dehydroxylation at $600{ }^{\circ} \mathrm{C}\left(\operatorname{larger} T_{2}{ }^{\text {micro }}(S D=1)\right)$ induced no $V_{\text {micro }}$ decrease (Figure 4) suggests that the loss of hydrophilicity had a lesser effect on the water access to the microporosity. Hence, the micropore volume reduction would be the main responsible for the $V_{\text {micro }}$ decrease.

In conclusion, the suitability of low-field ${ }^{1} \mathrm{H}$ NMR transverse relaxometry to directly detect and quantify microporosity is demonstrated on Stöber spheres, which exhibit intricate inner structure of elusive characterization. Colloidal crystal samples with known interparticle void configuration were used to avoid ambiguity in the micropore water discrimination and facilitate straightforward volume calculation. Relaxation experiments readily showed the presence of considerable micropore volume with high water-accessibility, in agreement with recent adsorption results. It was found that, upon drying under ambient conditions, the water-filled micropores were spontaneously evacuated - partially- but only after the voids were emptied. Thermal effects on the microporosity were easy to monitor by the relaxation mode changes, revealing that annealing at $>600{ }^{\circ} \mathrm{C}$ was needed to initiate micropores collapse, which was virtually accomplished at $900{ }^{\circ} \mathrm{C}$. Dehydroxylation of the inner micropore walls was already observed at $600^{\circ} \mathrm{C}$. Rather surprisingly, no significant effect of the progressive micropore hydrophobicity was observed on the water accessibility or drying. The use of NMR relaxometry for direct microporosity study is extendable to any multiporous system -in the common case of undefined meso- and macroporosity, complementary characterization by e.g. mercury intrusion or pycnometry would then be required for absolute quantification of the microporosity.

\section{Experimental Section}

Materials Stöber silica monodisperse spheres were purchased from Microparticles $\mathrm{GmbH}$ (nominal sizes $D=320$ and $260 \mathrm{~nm},<3 \%$ polydispersity). Massive, free-standing colloidal crystals were fabricated by slow sedimentation of the monodisperse spheres and drying under normal laboratory conditions ( $\left.35 \% \mathrm{RH}, 22^{\circ} \mathrm{C}\right)$. The controlled sedimentation process led to a densely-packed spheres assembly approaching an ordered fcc arrangement, as it was recognizable by the characteristic structural color of ordered artificial opals ${ }^{[8 a]}$ and confirmed by the observation of the corresponding Bragg peak with optical spectroscopy (Ocean Optics 2000+). Inspection by scanning electron microscopy (FEI Nova NANOSEM $230)$ verified the spheres arrangement into an fcc packing. A better ordering was found in the 320-nm CC. Some pieces of the asprepared samples were thermally annealed in air at different 
temperatures $\left(600,700,800\right.$ and $\left.900^{\circ} \mathrm{C}\right)$ in a conventional oven by slow heating $\left(1^{\circ} \mathrm{C} / \mathrm{min}\right)$ up to the nominal annealing temperature, waiting for $3 \mathrm{~h}$ and cooling $\left(10^{\circ} \mathrm{C} / \mathrm{min}\right)$ down to room temperature $\left(25^{\circ} \mathrm{C}\right)$.

NMR experiment ${ }^{1} \mathrm{H}$ NMR transverse relaxometry was performed on imbibed (fully saturated) samples and during subsequent drying. Each sample was immersed in water for 48 hours at ambient temperature and low-pressure conditions -to facilitate evacuation of trapped air bubbles from the pores- in order to reach full saturation $(S D=1)$. Desaturation down to $S D_{a}$ was achieved by slow, spontaneous water evaporation from the sample in the NMR tube. Lower $S D$ values were attained by further drying the sample on a hot plate $\left(120^{\circ} \mathrm{C}\right)$ or under high vacuum plus heating $\left(120^{\circ} \mathrm{C}\right)$, measuring immediately after that. CarrPurcell-Meiboom-Gill (CPMG) experiments, performed by a MINISPEC MQ20 (Bruker, Germany), consisted of a serial of $180^{\circ}$ radiofrequency (RF) pulses following a first $90^{\circ}$ pulse, with constant time interval between the $180^{\circ}$ pulses (echo time interval). Echo trains were obtained by recording the signal amplitude in the middle time between $180^{\circ}$ pulses. The CPMG pulse sequence compensates for the background gradients and allows fast recording of the echo train with amplitudes attenuated by transverse relaxation phenomena. The distribution of relaxation

[1] M. Thommes, K. Kaneko, A. V. Neimark, J.P. Olivier, F. Rodriguez-Reinoso, J. Rouquerol, K.S.W. Sing. Physisorption of gases, with special reference to the evaluation of surface area and pore size distribution (IUPAC Technical Report). Pure Appl.Chem. 2015, 87, 1051-1069.

[2] a) R. Kimmich, NMR: tomography, diffusometry, relaxometry, Springer, Berlin, 1997. b) J. P. Korb. Nuclear magnetic relaxation of liquids in porous media. New J. Phys. 2011, 13, 035016.

[3] a) T. R. Todoruk, C. H. Langford, A. Kantzas. Pore-Scale Redistribution of Water during Wetting of Air-Dried Soils As Studied by Low-Field NMR Relaxometry. Environ. Sci. Technol. 2003, 37, 2707-2713. b) V. Bortolotti, R. J. S. Brown, P. Fantazzini, M. Mariani. Evolution of a short-T2 liquid-like $1 \mathrm{H}$ signal during the hydration of white portland cement. Microporous Mesoporous Mater. 2013, 178, 108-112. c) A. Bede, A. Scurtu, I. Ardelean. NMR relaxation of molecules confined inside the cement paste pores under partially saturated conditions. Cem. Concr. Res. 2016, 89, 56-62. d) M. Meyer, C. Buchmann, G. E. Schaumann. Determination of quantitative pore-size distribution of soils with ${ }^{1}$ H NMR relaxometry. Eur. J. Soil Sci. 2018, 69, 393406.

[4] O. V. Petrov, I. Furó. NMR cryoporometry: Principles, applications and potential. Prog. Nucl. Magn. Reson. Spectrosc. 2009, 54, 97-122.

[5] W. Stöber, A. Fink, E. Bohn. Controlled Growth of Monodisperse Silica Spheres in the Micron Size Range. J. Colloid Interface Sci. 1968, 26, 62-69.

[6] a) M. Yang, H. Wu, H. Wu, C. Huang, W. Weng, M. Chen, H. Wan. Preparation and characterization of a highly dispersed and stable $\mathrm{Ni}$ catalyst with a microporous nanosilica support. RSC Adv. 2016, 6, 81237-81244. b) N. Sahiner, A. O. Yasar. A new application for colloidal silica particles: natural, environmentally friendly, low-cost, and reusable catalyst material for $\mathrm{H}_{2}$ production from $\mathrm{NaBH}_{4}$ methanolysis. Ind. Eng. Chem. Res. 2016, 55, $11245-11252$

[7] a) A. Meddahi-Pell, A. Legrand, A. Marcellan, L. Louedec, D. Letourneur, L. Leibler. Organ Repair, Hemostasis, and In Vivo Bonding of Medical Devices by Aqueous Solutions of Nanoparticles. Angew. Chem. Int. Ed. 2014, 53, 6369 -6373. b) T. L. Moore, D. Hauser, T. Gruber, B. Rothen-Rutishauser, M. Lattuada, A. Petri-Fink, R. Lyck. Cellular shuttles: monocytes/macrophages exhibit transendothelial transport of nanoparticles under physiological flow. ACS Appl. Mater. Interfaces 2017 9, 18501-18511.

[8] a) F. Gallego-Gómez, A. Blanco, C. López. Exploration and Exploitation of Water in Colloidal Crystals. Adv. Mater. 2015, 27, 2686-2714. b) Y. Zhang, $\mathrm{Q}$. Fu, J. Ge. Photonic sensing of organic solvents through geometric study of dynamic reflection spectrum. Nature Commun. 2015, 6, 7510. c) F. Gallego-Gómez, M. Morales, A. Blanco, C. López. Tunable Visual Detection of Dew by Bare Artificial Opals. Adv. Funct. Mater. 2018, 28, 1800591. times, extracted from the CPMG echo train by numerical ILT must be, however, analyzed with caution, as the ILT is ill-conditioned and may be affected by noise. ${ }^{[16 c]}$ Low proton resonance frequency (20 $\mathrm{MHz}$ ) and short echo time interval (80 $\mu$ s) were used to reduce diffusion effects on echo train attenuation; ${ }^{[15 a]}$ short recycle delay $(0.5 \mathrm{~s})$ and small number of scans (512) were further chosen to minimize water evaporation (and consequent $S D$ fluctuations) during the measurement. Due to the larger relaxation times in annealed samples, the recycle delay was increased to $5 \mathrm{~s}$ in order to wholly account the spin relaxation and correctly quantify the full-saturation slow modes.

\section{Acknowledgments}

This work was supported by the Spanish MINECO project MAT2014-58731-JIN and MCIU grant RTI2018-093921-B-C41. I. A acknowledges the partial support of the project 21 PFE in the frame of the program PDI-PFE-CDI 2018.

Keywords: microporosity; confined water; ${ }^{1} \mathrm{H}$ low-field NMR transverse relaxometry; Stöber silica spheres; porous media

[9] a) L. Fan, D. P. Josephson, A. Stein. Colloidal assembly: the road from particles to colloidal molecules and crystals. Angew. Chem. Intern. Ed. 2011, 50, 360-388. b) F. Gallego-Gómez, M. Ibisate, D. Golmayo, F. J. Palomares, M. Herrera, J. Hernández, S. I. Molina, A. Blanco, C. López. Light emission from nanocrystalline Si inverse opals and controlled passivation by atomic layer deposited $\mathrm{Al}_{2} \mathrm{O}_{3}$. $\mathrm{Adv}$. Mater. 2011, 23, 5219. c) Z. Teng, S. Wang, X. Su, G. Chen, Y. Liu, Z. Luo, W. Luo, Y. Tang, H. Ju, D. Zhao, G. Lu. Facile synthesis of yolk-shell structured inorganicorganic hybrid spheres with ordered radial mesochannels. Adv. Mater. 2014, 26, 3741-3747.

[10] J. Farrando-Pérez, C. López, J. Silvestre-Albero, F. Gallego-Gómez. Direct Measurement of Microporosity and Molecular Accessibility in Stöber Spheres by Adsorption Isotherms. J. Phys. Chem. C 2018, 122, 22008-22017.

[11] a) J. P. Gallas, J. C. Lavalley, A. Burneau, O. Barres. Comparative study of the surface hydroxyl groups of fumed and precipitated silicas. 4. Infrared study of dehydroxylation by thermal treatments. Langmuir 1991, 7, 1235-1240. b) A Labrosse, A. Burneau. Characterization of Porosity of Ammonia Catalysed Alkoxysilane Silica. J. Non-Cryst. Solids 1997, 221, 107-124. c) F. Gallego-Gómez, V. Morales-Flórez, M. Morales, A. Blanco, C. López. Colloidal crystals and water: Perspectives on liquid-solid nanoscale phenomena in wet particulate media. Adv. Colloid Interface Sci. 2016, 234, 142-160.

[12] L. T. Zhuravlev. The surface chemistry of amorphous silica. Zhuravlev model. Colloids Surf. A 2000, 173, 1-38.

[13] a) M. D. Sacks, T.-Y. Tseng. Preparation of $\mathrm{SiO}_{2}$ Glass from Model Powder Compacts: I, Formation and Characterization of Powders, Suspensions, and Green Compacts. J. Am. Ceram. Soc. 1984, 67, 526-532. b) E. N. Samarov, A. D. Mokrushin, V. M. Masalov, G. E. Abrosimova, G. A. Emel'chenko. Structural Modification of Synthetic Opals during Thermal Treatment. Phys. Solid. State 2006, 48, 1280-1283.

[14] S. Meiboom, D. Gill. Modified spin-echo method for measuring nuclear relaxation times. Rev. Sci. Instrum. 1958, 29, 688-691.

[15] a) J. Mitchell, T. C. Chandrasekera, L. F. Gladden. Obtaining true transverse relaxation time distributions in high-field NMR measurements of saturated porous media : Removing the influence of internal gradients. J. Chem. Phys. 2010 132, 244705-244710. b) S. Muncaci, S. Boboia, I. Ardelean. The effect of diffusion in internal gradients on nuclear magnetic resonance transverse relaxation measurements. AIP Conf. Proc. 2013, 1565, 133-136.

[16] a) S. W. Provencher. CONTIN: A general purpose constrained regularization program for inverting noisy linear algebraic and integral equations. Comput. Phys. Commun. 1982, 27, 229-242. b) L. Venkataramanan, Y. Song, M. D. Hürlimann. Solving Fredholm integrals of the first kind with tensor product structure in 2 and 2 . 5 dimensions. IEEE Trans. Signal Process. 2002, 50, 1017-1026. c) G. C. Borgia, R. J. S. Brown, P. Fantazzini. Uniform-penalty inversion of multiexponential decay data - II. Data spacing, T-2 data, systematic data errors, and diagnostics. J. Magn. Reson. 2000, 147, 273-285. 
[17] M. Simina, R. Nechifor, I. Ardelean. Saturation-dependent nuclear magnetic resonance relaxation of fluids confined inside porous media with micrometer-sized pores. Magn. Reson. Chem. 2011, 49, 314-319.

[18] In S2-0 ( $D=260 \mathrm{~nm}$, with void internal size of 59-108 nm), it is found that $T_{2}{ }^{\text {void }} / T_{2}$ micro $(S D=1)=24$, so that Eq. (1) leads to a small porosity of $2-5 \mathrm{~nm}$.

[19] A minor mesoporous volume -with small pore size of 2-3 nm-may also be present in Stöber spheres. ${ }^{[10]}$ However, compared to the microporosity, its low relative fraction and similar pore size would make its contribution in discernible but lead to a broadening -and a slight asymmetry- of the fast relaxation mode.

[20] In an ideal close-packed fcc unit cell, $A^{\text {void }}=0.26$ (in volume fraction of the cell) and $A^{\text {micro }}=0.74 V_{\text {micro }}$ (being $V_{\text {micro }}$ here expressed in volume fraction of the sphere). Hence, $V_{\text {micro }}=0.26 / 0.74 A^{\text {micro }} / A^{\text {void }}$. Any degree of disorder in the CC sample implies a somewhat larger void volume, which would lead to some underestimate of $V_{\text {micro }}$. Thus, a realistic void volume of $30 \%$ in our samples would yield a microporosity of $19-20 \%$ of the sphere volume, stil in good agreement with Ref. [10]. (The slight excess could be explained by the minor contribution of the sphere inner mesopores, which is not accounted in the methodology used in Ref. [10]; or by a greater water access to the inner sphere volume under our soaking conditions - note that a total micropore volume of ca. $20 \%$ was obtained in Ref. [10])

[21] After sample outgassing, water is re-adsorbed up to $S D_{a}=0.13$ when the equilibrium under ambient conditions is recovered, a fact that discards spurious contributions, such as remaining water from sphere synthesis or CC fabrication.

[22] For example, by increasing the tube temperature from 35 to $60 \cong \mathrm{C}$, a clear decrease in the micropore water content $\left(A^{\text {micro }}\right)$, down to about one-third, was measured.

[23] a) F. Gallego-Gómez, A. Blanco, C. López. In situ optical study of water sorption in silica colloidal crystals. J. Phys. Chem. C 2012, 116, 18222-18229. b) H. Yang, P. Jiang, B. Jiang. Vapor detection enabled by self-assembled colloidal photonic crystals. J. Colloid Interface Sci. 2012, 370, 11-18. c) F. Gallego-Gómez, M. Morales, A. Blanco, C. López. Bare Silica Opals for Real-Time Humidity Sensing. Adv. Mater. Technol. 2019, 4, 1800493.

[24] F. Gallego-Gómez, A. Blanco, V. Canalejas-Tejero, C. López. Water-dependent photonic bandgap in silica artificial opals. Small 2011, 7, 1838-1845.

[25] a) A. A. Chabanov, Y. Jun, D. J. Norris. Avoiding Cracks in Self-Assembled Photonic Band-Gap Crystals. Appl. Phys. Lett. 2004, 84, 3573-3575. b) S. Romeis, J. Paul, M. Hanisch, V. R. R. Marthala, M. Hartmann, R. N. K. Taylor, J. Schmidt, W. Peukert. Correlation of Enhanced strength and internal structure for heat treated submicron Stöber silica particles. Part. Part. Syst. Charact. 2014, 31 664-674.

[26] F. Gallego-Gómez, A. Blanco, D. Golmayo, C. López. Three regimes of water adsorption in annealed silica opals and optical assessment. Langmuir 2011, 7, 13992. 